\title{
Advances in Smart and Intelligent Multimedia Platforms for Pervasive Computing
}

\author{
Ken Choi • Haiqing Nan • Wook Choi
}

Published online: 17 February 2015

(C) Springer Science+Business Media New York 2015

Recent advances in various computing and telecommunication fields, along with the proliferation of multimedia-capable mobile devices, such as laptops, portable media players, personal digital assistants, and smart phones, have stimulated the development of pervasive multimedia services/applications. These upcoming services and applications essentially require a smart and intelligent platform that is able to provide noble development and operation tools for accommodating the pervasive system, applications, and services. This special issue presents original research papers addressing research challenges in all areas of smart and intelligent multimedia platform for pervasive systems, applications, and services. This issue consists of eleven papers, which are briefly discussed as follows.

The openness of the Android system allows user to choose the various online markets. Since such openness also invokes critical threats, such as malware and illegal use, several markets have deployed strong authentication techniques. However, such methods are also an inconvenience to legal users. Therefore, in "Authentication of Mobile Application through Various Local Distributors"(10.1007/s11042-013-1520-y), the author proposes the concept of an authentication model that makes purchased application available in most cases.

The paper entitled "An interactive pervasive whiteboard based on MVC architecture for ubiquitous collaboration"(10.1007/s11042-013-1458-0) presents the whiteboard application on 3G and Wi-Fi Android platform based mobile devices, based on MVC (Model View Controller) architecture for ubiquitous collaboration and also presents interaction and usability study with multiple users using small-sized devices, mid-sized devices, and large-sized device to show how disparate access devices and networking have an effect on software design of shared applications in an ubiquitous collaboration system.

In "Network Security Camera System and its Application for Consumer Electronics in Ubiquitous Environment"(10.1007/s11042-013-1442-8), the authors present a network security camera system and its application to provide visual information to consumer electronics devices in an ubiquitous

K. Choi $(\square)$

Department of ECE Illinois Institute of Technology, Chicago, IL 60616, USA

e-mail: kchoi.jopt@gmail.com

H. Nan

Intel China Research Lab Haidian District, Beijing, China 100080

W. Choi

Samsung Research Center, Yongin, Suwon, South Korea 443-749 
environment. To demonstrate the applicability of the network security system to consumer electronics devices, authors applied the proposed system to a vacuum cleaner.

The "Adaptive multi-channel allocation for vehicular infrastructure mesh systems"(10.1007/s11042-013-1752-x) paper focuses on a wireless solution for vehicular infrastructure systems. Wireless mesh networks solutions cannot appropriately support various vehicular applications that require high rate and low latency communications. Therefore, the authors present design of an adaptive multi-channel allocation for vehicular infrastructure mesh systems and performance evaluation is conducted by the QualNet 5.0 simulator.

The paper entitled "Segmentation-based view synthesis for multi-view video plus depth"(10.1007/s11042-013-1747-7) presents a novel view synthesis algorithm for three dimensional video. The proposed algorithm is based on segmentation using the multi-level thresholding method and experimental results show that the proposed methods achieve an average PSNR gain of $0.98 \mathrm{~dB}$ for the multi-view test sequences and also improve the subjective quality of the synthesized views.

In "Multi-Hop-based Opportunistic Concurrent Directional Transmission in $60 \mathrm{GHz}$ WPANs"(10.1007/s11042-014-1959-5) paper, authors propose a multi-hop-based opportunistic concurrent directional transmission scheme for the directional multicast communication where the relay mechanism is generated depending on the locations of multicast users to maximize the sum rate. Extensive simulation results demonstrate that the M-OCDT scheme can improve the average overall throughput from 81 to $89 \%$ compared with the conventional non-relay directional multicast procedure.

To maintain the data security and integrity, one of the main researches is information hiding for images. The paper "Data hiding of High Compression Ratio in VQ Indices with Neighboring Correlations"(10.1007/s11042-014-2019-x) proposes a data hiding method on the indices of VQ compression. The proposed data hiding can enhance the embedding capacity and bit rate of encoding and also can use the output bit stream to reconstruct the cover image.

The security of web browsers has become an increasingly important issue in recent years. In "Web Security in a Windows System As PrivacyDefender in Private Browsing Mode"(10.1007/s11042-014-2003-5) paper, authors design a mechanism in Windows XP that observes the behaviors and patterns related to the creation and deletion of files in Firefox while in private browsing mode. The authors then focus on the files which were not deleted, and cleared them by means of anti-forensics manners. In other words, web browsers can be made comprehensively secure with this mechanism.

Criminal profiling such as behavioral and investigative data profiling could help to identify the criminals leaking the secrets. The application of criminal profiling may contribute to prevent and reduce industrial espionage. The paper entitled "Criminal Profiling and Industrial Security"(10.1007/s11042-014-2014-2), is a study that attempts to apply criminal profiling to industrial security to eventually prevent and control industrial espionage.

In "Hybrid Storage-based Caching Strategy for Content Delivery Network Services"(10.1007/ s11042-014-2215-8), the authors propose a novel caching scheme for a content delivery network. A caching scheme, in which the first part of the frequently referenced content is stored in a solid state drive while the rest of the video content is stored in hard disk drive, is proposed. Performance can be significantly improved with low extra cost in a cache server of content delivery network.

The need for lightweight cryptography is on the rise as the transition has been made from wired to wireless network. Since Signcryption combines the signature and encryption, the cost is much less than many Signcryption schemes proposed until now. The paper entitled "An Efficient Signcryption scheme with forward secrecy and public verifiability based on hyper elliptic curve cryptography"(10.1007/s11042-014-2283-9) highlights limitations of the existing ECC based schemes using Signcryption and further proposes an efficient lightweight Signcryption scheme based on 
HECC. The scheme reduced significant amounts of computation, communication costs and message size as compared to existing Signcryption schemes.

These eleven papers cover a wide range of multimedia tools and applications in terms of smart and intelligent multimedia platforms for pervasive systems, applications and services. We hope that this special issue will prove to be a valuable resource for researchers and practitioners and would like to show our appreciation to all the authors who submitted papers to this special issue. 